\title{
Malnutrition Intervention in Low Socioeconomic Senior Populations
}

\author{
Taryn Vanderberg
}

\section{ABSTRACT}

This study aims to identify the effectiveness of malnutrition intervention programs within senior populations. Government subsidized nutrition intervention programs, such as Meals on Wheels, play a vital role in the prevention of malnutrition in lower socioeconomic senior populations in the United States (Roy, 2006). For many older adults, meals received via nutrition programs serve as a lifeline, meeting essential nutritional needs and preventing premature institutionalized care (Lepore, 2019). Sixty-three Meals on Wheels (MOW) participants residing in Southern California were assessed, comparing nutritional status upon program intake against nutritional status after three to six months to identify improvement or decline. This study relied on self-reporting on the part of senior participants to explore the characteristics related to socioeconomic status and nutritional risk, and collect quantitative data. Further, it aimed to highlight whether nutritional risk was decreased through program usage. Access to the MOW nutrition program was found to correlate with a reduction in malnutrition risk among the participants in the study. Through the use of nutrition programs and their evaluations, malnutrition and malnutrition risk may be detected earlier, and subsequent measures for prevention can be employed. 


\section{Introduction}

Malnutrition encompasses the deficiencies, imbalances, or excesses of nutrients, which result in an adverse, measurable effect on one's body composition, function, or clinical outcome (Elia, 2000). The term addresses three categories, including under-nutrition (wasting, stunting, and underweight), micronutrient malnutrition (a lack or excess of minerals and vitamins), and obesity or diet-related disease (World Health Organization, 2020). Though its effects are primarily physiological, its causes are often systemic, with under-nutrition and micronutrient malnutrition affecting low income elderly populations at a disproportionate rate (Evans, 2005). With an estimated 9.8 million older adults currently at risk for malnutrition in the United States, and approximately 10,000 Americans predicted to turn 65 each day through 2050, atrisk senior populations will only increase (Eggsdorfer, 2018). Seniors, or older adults aged 60 and over, are now the fastest growing population base, estimated to increase by $3 \%$ annually over the next two decades (Eggsdorfer, 2018).

This study highlights the risk factors among vulnerable senior populations, using modified questions from the Mini Nutritional Assessment to collect relevant data (Nestle Nutrition Institute, 2009). The Mini Nutritional Assessment was created by Nestle Corporation, and is a validated screening tool used by government agencies and healthcare providers to predict nutritional outcomes and cost of care (Guigoz, 2002). Data collected from the population sample conveyed a correlation between socioeconomic conditions and malnutrition/malnutrition risk in individuals over 60 years of age, with socioeconomic variables such as marital status, education level, living alone, and local food costs strongly correlated to nutritional status. In general, research has found that for every income increase of $10 \%$ or more, malnutrition rates decline by up to $5 \%$, further solidifying the link between malnutrition and poverty (Haddad, 2003). Malnutrition should always be evaluated in the context of its social and economic contributors. These key factors allow for the identification of at-risk individuals, groups, and populations (Alderman et al., 2007).

\section{Malnutrition Risk and Consequences}

A predecessor for mortality, malnutrition leads to health consequences such as muscle wasting, reduced cardio-respiratory function, gastrointestinal function, delayed wound healing, and psychosocial effects (Saunders, 2010). Malnutrition affects all populations, with millions at risk daily across the globe (World Health Organization, 2020). Though it is often associated with low income countries, malnutrition remains prevalent in the United States, where 14.3 million U.S. households were food-insecure in 2017 ("Food Insecurity in U.S. Households," 2018). In the U.S. alone, 4.7 million people aged 65 and older currently live below the Federal Poverty Line (Dalaker, 2019).

It has been found that nutritional status directly impacts the rate of functional and physiological decline experienced with age (Blumberg, 1997). Therefore, malnutrition remains a critical public health concern for these populations. Elderly individuals with comorbidities such as respiratory disease, heart disease, and age-related frailty remain at an even higher risk (Schlip, 2011). Of Americans aged 65 and older, $16 \%$ consume fewer than 1000 calories daily (Wallace, 1999). Malnutrition risk includes factors such as old age, low socioeconomic status, and poor physical or mental health, which predispose individuals to food insecurity and improper nourishment (Bekkering, 2016). The rate of risk increases in those that are ill, homebound, have limited access to resources and/or medical care, or are living in poverty (Wallace, 1999). This risk ranges up to $50 \%$ higher for those hospitalized, and up to $60 \%$ for those in institutionalized facilities such as senior rehabilitation centers, where adequate nutrition upon discharge can be crucial to a successful recovery (Wallace, 1999).

Malnutrition carries an estimated annual cost of $\$ 51$ billion in the United States alone, creating a burden for the entire health care system (The Gerontological Society of America, 2015). As average individual life spans have increased over the past several decades, people in developed countries are now living for an average of 80 years (United Nations, 2012). This has led to an inevitable strain on resources to support both quality of life and adequate care, 
well into one's senior years (United Nations, 2012). Unfortunately, malnutrition and its associated risk factors impact this quality of life directly (Schlip, 2011).

Early intervention is the best method of curbing these risks, preventing further health consequences, while simultaneously, reducing burden to the system (Schlip, 2011). This can occur in a variety of ways, but common methods include government-subsidized intervention programs such as Meals on Wheels (Roy, 2006). For the purpose of this study, a MOW program was selected for examination.

\section{Meals on Wheels Program Background}

Funded in part through the Older Americans Act, Meals on Wheels programs operate across the country, with a collective goal of strengthening senior nutritional health and reducing morbidities (Roy, 2006). Nutritious meals are hand-delivered to homebound seniors who qualify via dedicated volunteers. The primary purpose of volunteer delivery is to provide meals, as well as a safety check and a friendly visit for those who are isolated. Programs are independent and locally run through non-profits, each using their own criteria in assessing nutritional risk, program need, and whether an individual qualifies to receive government subsidized service (Meals on Wheels America, 2020). Though private pay options exist, these nutrition intervention programs are utilized at local, state, and federal levels to collect data that determines funding, and both track and improve senior health and nutrition status in local communities. Qualifying programs receive partial funding through the Older Americans Act, Medicaid, and the United States Department of Agriculture (Meals on Wheels America, 2020).

According to Meals on Wheels America, $39 \%$ of the total amount spent through the Older Americans Act goes towards providing nutritious meals to 2.4 million seniors each year (Meals on Wheels America, 2020). The majority of seniors utilizing the program are deemed low income, unable to afford the food they need. In 2020, the organization announced it has served 21 million fewer meals compared to 2005, due to increases in food and transportation costs (Meals on Wheels America, 2020). Unfortunately, program funding has remained the same. Therefore, the welfare and nutritional health of at-risk senior populations remain in jeopardy.

\section{Participants}

This study drew from a population of MOW recipients residing in Southern California, who had been receiving MOW services for a period of three to six months. Data was collected between January 2020 and August of 2020, with the intention of using total population sampling. Program participants were informed how anonymous data would be collected and utilized, both for the program and for the purpose of this study. Individuals were able to decline if they did not wish to participate, and their results were omitted from the research pool. Initial intake assessments occurred both in person and via telephone (all follow-up assessments were conducted exclusively via telephone calls due to Covid-19). Subsequent follow-up evaluations occurred at three to six months. All recipients who agreed to data collection and had been on the program for three to six months were included in the study. Initial intake assessments determined participants' existing health and nutritional status prior to joining the program. Follow-up assessments examined malnutrition/malnutrition risk after time using the program, through reasking the same targeted intake questions about daily meals consumed, weight gain or loss, appetite, and current health conditions, noting any changes.

\section{Method}

Program participants received an initial assessment from a trained nutrition program manager, consisting of modified questions from the Mini Nutrition Assessment to identify nutritional status at intake. Questions were modified per the MOW program's own eligibility criteria, and therefore, did not include a body mass index (BMI) measurement. Participants were followed up with at three or six month intervals for further assessment, and asked the same questions to determine change in nutritional status and program outcomes.

Intake to a nutrition program such as MOW is typically two-step, involving a telephone questionnaire to assess risk and program need, as well as an additional home visit to verify the 
Characteristics of Participants

Percentage of Participants

\begin{tabular}{lc}
\hline Male & $38.09 \%$ \\
Female & $61.9 \%$ \\
Caucasian & $74.60 \%$ \\
Hispanic & $11.11 \%$ \\
Asian & $9.52 \%$ \\
African American & $3.17 \%$ \\
Other Race & $1.59 \%$ \\
Frail Physical Condition & $77.78 \%$ \\
Living Alone & $66.67 \%$ \\
Recently Hospitalized & $7.94 \%$ \\
Immobile or Wheel-Chair Bound & $3.17 \%$
\end{tabular}

Table 1. Characteristics of Participants.

health status and living arrangements of the participant. Demographics are collected as well as socioeconomic circumstances, health background, and an assessment of the senior's mobility. Modified questions from the Mini Nutrition Assessment are utilized to assess each client, with the exception of the BMI measurement. Responses are recorded on paper and kept on file for each individual client for the duration of their stay with the program. Follow-up assessments, using the same questions from the intake, are intended to track changes in nutritional status across time as well as measure successful program outcomes. For the purpose of this study, nutritional outcomes were evaluated by comparing the intake assessments against the three to six month assessments to track any change in nutritional status (note: due to Covid19, all program follow-up assessments were conducted via telephone, per state guidance).

\section{Participant Profile}

Upon the initial intake screening, $42.86 \%$ of participants were at a medium to high risk for malnutrition, $57.14 \%$ percent were at a low risk, and $100 \%$ of those accepted to the program were at risk for some level of malnutrition. Many exhibited characteristics of frailty and faced socioeconomic hardships such as being unable to afford food, being unable to secure three meals per day or eating minimal fruits, vegetables, and dairy products (micronutrient rich foods typically associated with a healthy diet). While receiving
MOW services, each participant received three balanced meals daily, including a pre-packaged breakfast, lunch, and dinner. These meals are planned by a licensed registered dietician to ensure the nutritional and caloric needs of adult seniors are met.

Out of the $63 \mathrm{MOW}$ participants in the study, 24 were males (38.09\%) while 39 were females $(61.9 \%)$. Of the sixty-three participants, 43 received meals five days a week, with the remainder receiving meals three days a week. Only nine participants received meals seven days a week due to a high level of need. The average age of participants was determined to be 82 . Forty-two participants (66.67\%) were found to be living alone, while 21 (33.33\%) resided with, at minimum, one other individual (spouse, family member, friend, or other). Forty-nine were determined to be frail $(77.78 \%)$, based on the program questionnaire and in-home assessment. General characteristics from all participants were compiled for reference and displayed in Table 1.

Health data, including recent hospital admissions, was collected upon intake and follow-up assessment, with five (7.94\%) having been recently hospitalized prior to or during use of the service. Three (3.17\%) were immobile or wheelchair bound, unable to access food through shopping or leaving the home. Of the assessed program participants, 47 identified as Caucasian $(74.60 \%)$,six identified as Asian (9.52\%), seven identified as Hispanic (11.11\%), two identified as 
African American (3.17\%), and one identified their race as Other (1.59\%).

Out of 105 initial assessments completed, 42 participants left the program prior to a three month assessment. Twelve (28.57\%) opted to end the service due to the Covid-19 pandemic, fearing contamination. Though state guidelines mandated contact-free delivery, these participants opted to rely on grocery delivery services, neighbors, or relatives for meals instead. Of the remaining participants who ended service prior to a follow-up assessment, 11 did so due to dislike of the provided meals $(26.19 \%)$, six discontinued due to hospital or skilled nursing facility admission (14.29\%), and two discontinued due to other unknown means of obtaining meals (4.76\%). Eleven participants (26.19\%) discontinued due to health conditions, requiring specialized dietary needs the program was unable to accommodate.

Of the remaining participants assessed, $36(57.14 \%)$ were at a low risk of malnutrition upon initial intake, while $27(42.86 \%)$ were at a medium to high risk. Upon follow-up, it was found there was a significant reduction in malnutrition risk for those in the program. According to the program's assessment criteria, 12 is the minimum score denoting nutritional risk, while 21 is the highest.

\section{Contributing Socioeconomic and Health Factors}

Of the participants, 42 (66.67\%) were single or widowed and reported living alone. Twenty-three participants $(36.50 \%)$ reported health issues that altered the type or amount of food they consumed daily, $14(22.22 \%)$ reported tooth, mouth, or swallowing issues that made it difficult to consume food. Four participants $(6.35 \%)$ reported depression or other neurological issues. Thirty-two (50.79\%) reported not having enough money to purchase needed food. Fifty-nine (93.66\%) reported being unable to get out to shop for food (due to decreased mobility or physical condition), 38 (60.32\%) reported being unable to cook or prepare meals (often due to an inability to stand for long periods, operate the stove, or use cooking utensils). One $(1.58 \%)$ reported being unable to feed themselves, requiring assistance from a spouse, family member, or professional caretaker. The health and economic profile of program participants is outlined in Table 2 below.

Federal Poverty Line reported being more likely to obtain food from other sources, including grocery delivery and other take-out food delivery services. Those below the Federal Poverty Line reported being unable to access these resources due to a lack of income. Of those who reported being below the Federal Poverty Line, $63.82 \%$ expressed interest in community food banks but were unable to access this resource due to being home-bound. This data, though preliminary, allows for enhanced understanding of the characteristics and socioeconomic status of seniors who access nutrition intervention programs.

Profile of Program Participants

Percentage Impacted

\begin{tabular}{lc}
\hline Health issues altering amount of food consumed daily & $36.50 \%$ \\
Tooth, mouth, or swallowing issues & $22.22 \%$ \\
Chronic depression, anxiety, dementia, or other neurological issues & $6.35 \%$ \\
Does not have enough money to purchase food needed & $50.79 \%$ \\
Unable to shop for food (due to mobility) & $93.66 \%$ \\
Unable to cook or prepare food (due to physical condition) & $60.32 \%$ \\
Unable to feed self (requires assistance) & $1.58 \%$ \\
Income above Federal Poverty Line & $25.39 \%$ \\
Income below Federal Poverty Line & $74.60 \%$ \\
Interest in food banks/resources, but unable to access & $63.82 \%$
\end{tabular}

Table 2. Health and Economic Profile of Program Participants. 


\section{Findings and Program Effectiveness}

Of the 63 participants who were assessed at intake and again at a three or six month follow-up to measure change, 58 participants (92.06\%) had improved their malnutrition or malnutrition risk after at least three months in the program, reducing their overall nutritional risk score by four or more points. Three participants had a reduction of two points $(4.76 \%)$, and two experienced no change (3.17\%).

According to the initial data collected through this study, nutritional baseline status improved between the initial intake and the three month follow-up, remaining stable between three and six months of program participation. Of the 63 participants assessed, $92.06 \%$ increased their overall nutritional status. Of those participants, 53 $(91.38 \%)$ went from consuming two or less meals per day to three full meals per day. Fifty-four $(93.01 \%)$ did not report weight loss during their time on the program.

Through the use of targeted programs, nutritional risk can be reduced, preventing malnutrition and the adverse affects associated with it. This leads to improvements in the overall physical, emotional, and social wellbeing of low socioeconomic elderly populations who otherwise have no means of accessing adequate meals (Endevelt et al., 2011). Further, clients requiring additional interventions (due to safety concerns, cleanliness, cognitive decline, or suspected abuse or neglect) are able to access assistance through licensed case managers and social workers. These services were not relevant to the nutritional improvements outlined, and therefore, were not explored in this study, though they remain a program benefit. Research has found dietary intervention through case management improves medical and nutritional status among senior populations, ultimately improving outcomes and supporting nutrition program effectiveness (Endevelt et al., 2011).

Though this study provides preliminary evidence that program utilization may lead to improved nutritional status, there is no proof that improved outcomes occurred solely due to program participation. Without further knowledge into each participant's individual background, health status, income, and other means of support such as family, friends, and access to other resources, organizations, or social services, the conclusion that improvements are fully credited to MOW cannot be drawn.

\section{Limitations}

Due to the outbreak of Covid-19 in the United States and the subsequent state restrictions surrounding program delivery, followup assessments could not be conducted face to face, and information was collected via telephone. Further, intake assessments, which normally occur in-home, were also conducted via telephone. This led to a potential margin of error wherein self-reported responses collected from participants may not be fully accurate. In assessing individual capabilities surrounding daily activities or frailty, many may self-report higher levels of independence and capability than may otherwise be observed by a trained professional (Knauper, 2016).

Other limitations included the lack of a control group; therein, causality was not assessed. The data collected was merely a baseline, utilized in determining program eligibility, and did not explore deeper contributing health factors that may contribute to malnutrition or risk, nor did the program measure weight or $\mathrm{BMI}$ at intake. In assessing recent weight gain or loss via assessment, it was up to the participant to relay that information to the nutrition program manager, which may have resulted in an estimation on the part of the individual being assessed. This may have resulted in in-accurate self-reporting. Further strengthening of data collection methods should be employed by the program to better determine both short and longterm nutritional outcomes.

Due to the location of the program and the high-income suburban areas served, participants in the pool skewed predominantly Caucasian. Lastly, only 63 participants were evaluated; thus, conclusions cannot be accurately applied to the entire program or population. Larger and more diverse population samples should be sought in order to better assess outcomes and relevance as well as to reduce sample bias. 


\section{Conclusions}

The aim of this research was to examine malnutrition risk, its association with socioeconomic, health, and behavioral factors among senior populations, and outcomes related to intervention methods employed. Intervention programs provide seniors with needed nutritional support, leading to improvements in nutritional status, as well as access to resources such as case management, nutrition education materials, and social contact; all of which are crucial in promoting physical, mental, and emotional health and well-being among the elderly (McReynolds, 2004).

Though this study does not directly link causality to the program, it has been found that nutrition programs, such as MOW, appear to be of benefit in preventing malnutrition for low socioeconomic senior populations, otherwise unable to obtain resources and food. Malnutrition in elderly populations can continue to be prevented through early intervention and by providing nutritional support for those who are low income, isolated, or homebound due to frailty, immobility, or other health conditions (Guigoz, 2002). Malnutrition and its associated risk factors should continue to be a subject of research in order to identify and enhance similar programs, initiatives, and intervention strategies.

\section{References}

Alderman, H., Behrman, J., \& Hoddinott, J. (2007). Economic and nutritional analyses offer substantial synergies for understanding human nutrition. The Journal of Nutrition, 137(3), 537544.

Bekkering, G., Declercq, A., Duyck, J., FávaroMoreira, N., Krausch-Hofmann, S., Matthys, C., Vereecken,C., \& Vanhauwaert, E. (2016). Risk Factors for malnutrition in older adults: A systematic review of the literature based on longitudinal data. Advances in Nutrition, 7(3), 507-522. https://doi.org/10.3945/an.115.011254

Blumberg, J. (1997). Nutritional needs of seniors. Journal of the American College of Nutrition, 16(6), 517-523.

Dalaker, J., \&Li, Z. (2019). CRS Report: Poverty among Americans aged 65 and older.
Congressional Research Service. [Fact Sheet]. https://fas.org/sgp/crs/misc/R45791.pdf

Elia, M. (2000). Guidelines for detection and management of malnutrition. The Malnutrition Advisory Group, Standing Committee of BAPEN. https://doi.org/10.1046/j.1467$\underline{3010.2001 .00111 . x}$

Eggersdorfer, M., Akobundu, U., Bailey, R., Beaudreault, A., Bergeron, G., Blancato, R., Blumberg, J., Bourassa, M., Gomes, F., Jensen, G., Johnson, M., Mackay, D., Marshall, K., Meydani, S., Shlisky, J., \& Tucker, K. (2018). Hidden hunger: Solutions for America's aging populations. Nutrients, 10(9), 1210. https://doi.org/10.3390/nu10091210

Endevelt, R., Lemberger, J., Bregman, J., Kowen, G., Berger-Fecht, I., Lander, H., \& Shahar, D. R. (2011). Intensive dietary intervention by a dietitian as a case manager among community dwelling older adults. The Journal of Nutrition, Health \& Aging, 15(8), 624-630.

Evans, C. (2005). Malnutrition in the elderly: Amultifactorial failure to thrive. The Permente Journal, 9(3), 38-41. https://doi.org/10.7812/TPP/05-056

Guigoz, Y., Lauque, S., \&Vellas, B. (2002). Identifying the elderly at risk for malnutrition: The mini nutritional assessment. Clinical Geriatric Medicine, 18(4), 737-757. https://doi.org/10.1016/s0749-0690(02)00059-9

Haddad, L., Alderman, H., Appleton, S., Song, L., \& Yohannes, Y. (2003). Reducing child malnutrition: How far does income growth take us? World Bank Economic Review, 17(1), 107131.

Knauper, B., Carriere, K., Chamandy, M., Rosen, N., Schwarz, N., \& Xu, Z. (2016). How aging affects self-reports. European Journal on Ageing, 13(2), 185-193. https://doi.org/10.1007/s10433$\underline{016-0369-0}$

Lepore, M., \& Rochford, H. (2019). Addressing food insecurity and malnourishment among older adults: The critical role of Older Americans Act nutrition programs. Public Policy \& Aging Report, 29(2), 56-61. https://doi.org/10.1093/ppar/prz003 
McReynolds, J., \&Rossen, E. (2004). Importance of physical activity, nutrition, and social support for optimal aging. Clinical Nurse Specialist, 18(4), 200-206. https://doi.org/10.1097/00002800200407000-00011

Nestle Nutrition Institute. (2009). Mini Nutritional Assessment. Société des Produits Nestlé. https://www.mna-

elderly.com/forms/mini/mna mini english.pdf

Roy, M., \& Payette, H. (2006). Meals-on-wheels improves energy and nutrient intake in a frail freeliving elderly population. Journal of Nutrition Health and Aging, 10(6), 554.

Saunders, J., \& Smith, T., (2010). Malnutrition: Causes and consequences. Clinical Medicine Journal: Royal College of Physicians, 10(6), 624627. https://doi.org/10.7861/clinmedicine.10-6$\underline{624}$

Schilp, J., Wijnhoven, H., Deeg, D., \& Visser, M. (2011). Early determinants for the development of undernutrition in an older general population: Longitudinal aging study. British Journal of Nutrition, 106(5), 708-717.
The Gerontological Society of America National Academy on an Aging Society. (2015). Profiles of an aging society. [Fact Sheet]. https://www.geron.org/images/gsa/malnutrition/ malnutritionprofile.pdf

United Nations Population Fund (UNFPA) \& HelpAge International. (2012). Aging in the twenty-first century: $A$ celebration and a challenge. [Fact Sheet] https://www.unfpa.org/sites/default/files/pubpdf/Ageing\%20report.pdf

United States Department of Agriculture. (2018). Food security status of U.S. households in 2018. Economic Research Service, USDA. https://www.ers.usda.gov/topics/food-nutritionassistance/food-security-in-the-us/key-statisticsgraphics.aspx

Wallace, J. (1999). Principles of geriatric medicine and gerontology (4th ed.). McGraw-Hill. World Health Organization. (2020). Malnutrition. [Fact Sheet]. https://www.who.int/newsroom/fact-sheets/detail/malnutrition 